\title{
Acoustic Tamper Detection Sensor with Very Low False Alarm Rate
}

\author{
Lawrence Muijlwijk, and Jonathan Scott \\ Department of Engineering, The University of Waikato \\ Private Bag 3105, Hamilton, 3240, New Zealand \\ Email: jbs@waikato.ac.nz
}

\begin{abstract}
We describe a device designed to detect interference (vandalism or tampering) by acoustic means. The design employs both a piezoelectric vibration transducer and a common microphone in a novel mechanical arrangement. In contrast to conventional acoustic sensors that simply respond to vibrations above a threshold, this design analyses the outputs of the two to trigger an alarm. The method confers a near-zero susceptibility to triggering by external loud noises. No complex calculations are required so that only a low-cost, embedded microcontroller is required and the whole sensor can be very cheap. Extreme sensitivity can be achieved with little risk of false alarms.
\end{abstract}

\section{INTRODUCTION}

Tamper detection systems based on acoustic vibration have been used to detect interference with vehicles, fences, doors, gates, and enclosures for many decades. [1], [2] A piezoelectric transducer, alternately called a "shock sensor" or "resonance microphone", is often used to pick up the sound. The main problem with this type of system is a high false alarm rate. The basic system triggers when peak or averaged energy exceeds a threshold, and external events can convey more energy than some interference events, so that the selection of the threshold level becomes a tradeoff between sensitivity and susceptibility to false alarms. A well-known manifestation of this is car alarms going off because of a thunder clap or similar loud noise. Various schemes have been employed over the years to differentiate between geniune events and false alarms, both analog and using considerable DSP capability. [3]-[5] These transducers are now mostly confined to glass breakage applications, where the intensity and spectrum of the event makes it relatively easy to distinguish from ambient sounds so that false alarms are minimised. [6], [7]

A number of schemes have appeared that effectively try to minimise false alarms by dynamically adjusting the detection threshold, for example as a funcion of weather [8] or sound measured elsewhere [9]. Our solution resembles these approaches, but contains two transducers within a single small unit.

\section{The Proposed Design}

We note that a physical impact on the object to which a sensor is attached tends to transfer a lot more energy to the sensor as opposed to an ambient loud noise. This is because the sound waves travel much more efficiently in a solid material. Our design consists of a piezoelectric transducer connected to a baseplate and a second microphone that is acoustically

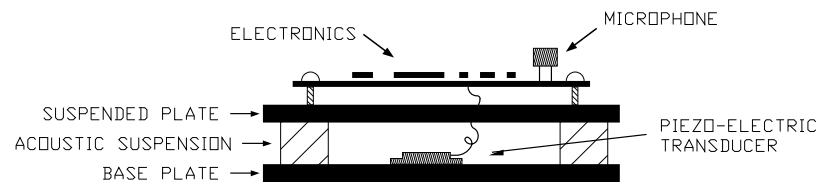

Fig. 1. Mechanical layout of the sensor. The piezoelectric transducer detects sound in the object to which the sensor is attached. The upper microphone detects sound in the environment. The acoustic suspension material provides acoustical resistance and forms a low-pass filter in conjunction with the mass of the suspended plate and circuit board, so that the upper microphone does not receive sound from the baseplate.

isolated. The arrangement is shown in figure 1. The acoustic suspension material provides acoustical resistance and forms a low-pass filter in conjunction with the mass of the suspended plate and circuit board, so that the upper microphone does not efficiently receive sound from the baseplate. [10] Simultaneously the two plates and the suspension material enclose the lower transducer so that it is not directly exposed to ambient sound.

The signals from the two transducers are amplified, filtered and fed to an embedded microcontroller. The controller is a MicroChip PIC12F683. This samples each of the two channels at $8 \mathrm{ksps}$ with 10-bit resolution. The running average energy in each channel is calculated in real time and the ratio of the energies is used to authenticate an alarm condition. The circuit requires only a voltage regulator, a dual CMOS opamp, and the US $\$ 2$ microcontroller as its active compliment. The circuit is shown in figure 2 . The prototype is programmed to allow sensitivity to be adjusted using a potentiometer and a pushbutton switch. Three LEDs show the status of the device. The prototype has also been programmed to log its activity in non-volatile flash memory within the microcontroller.

\section{Detector Response}

Frequency repsonse tests were conducted to assess the effectiveness of the microphone assembly. Ideally the transducer that is fixed to the baseplate will have higher sensitivity than the suspended transducer to sounds propagated in the baseplate, while the suspended transducer will have higher sensitivity than the fixed transducer to sounds propagated in the air around the sensor.

Two frequency response tests were run, the first where the unit was isolated from all vibrations (and would therefore only listen to ambient noise) and the second test with the unit 


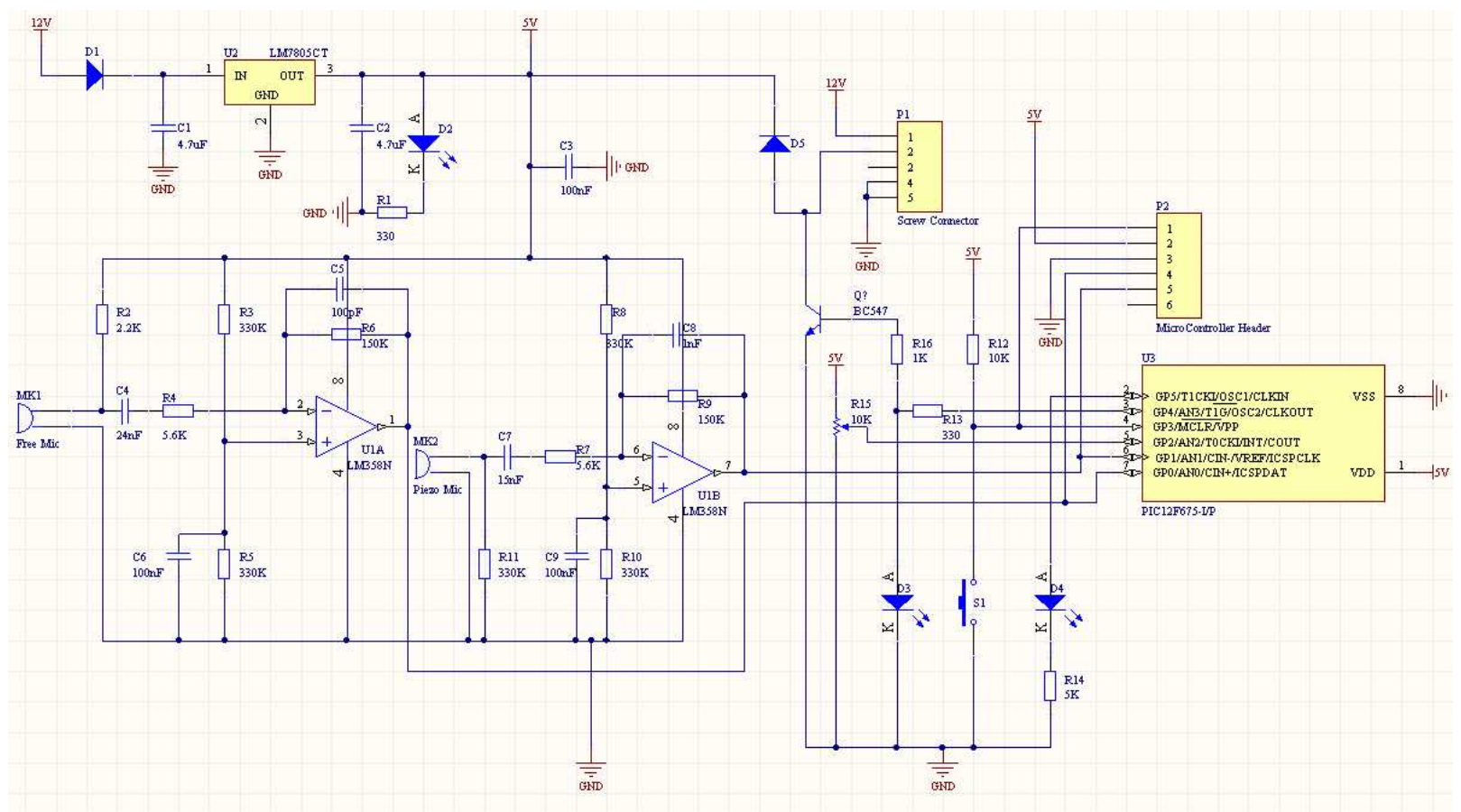

Fig. 2. Complete circuit diagram of the prototype sensor assembly.

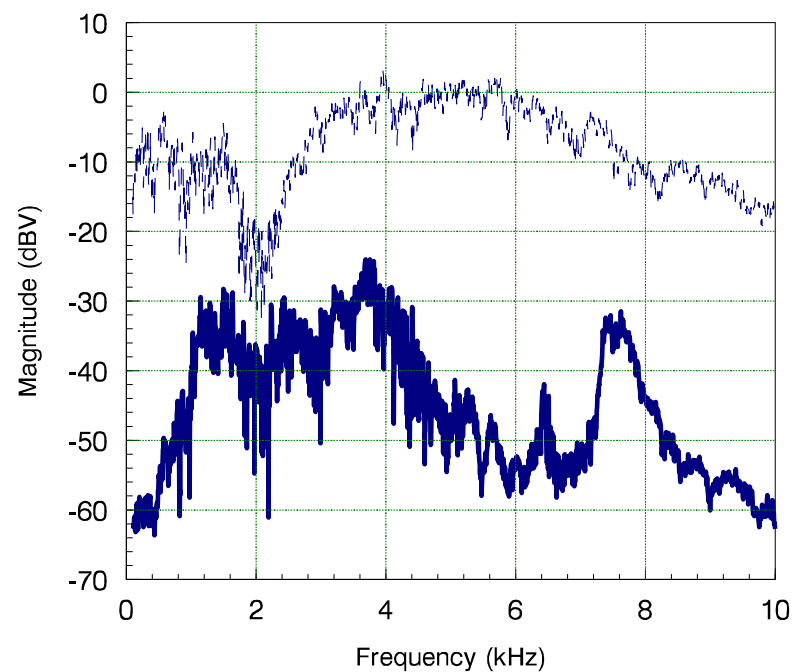

Fig. 3. Plot of the signal levels from the fixed and suspended transducers in response to vibrations in ambient air as a function of frequency. The solid, lower trace is the signal from the fixed piezoelectric transducer, the upper, light trace is the suspended microphone.

attached to a vibrating platform. Samples were taken in steps of $5 \mathrm{~Hz}$ from $100 \mathrm{~Hz}$ to $10 \mathrm{kHz}$. The first test shows remarkable results. Figure 3 plots the signal in the two channels. The free microphone signal exceeds that from the fixed unit at all frequencies. Figure 4 shows the reverse situation, where the sound is being generated by a transducer attached to the baseplate. In this case the fixed, piezoelectric transducer shows a higher sensitivity up to at least $6 \mathrm{kHz}$, but the traces cross over for a range of frequencies around $8 \mathrm{kHz}$. For this reason

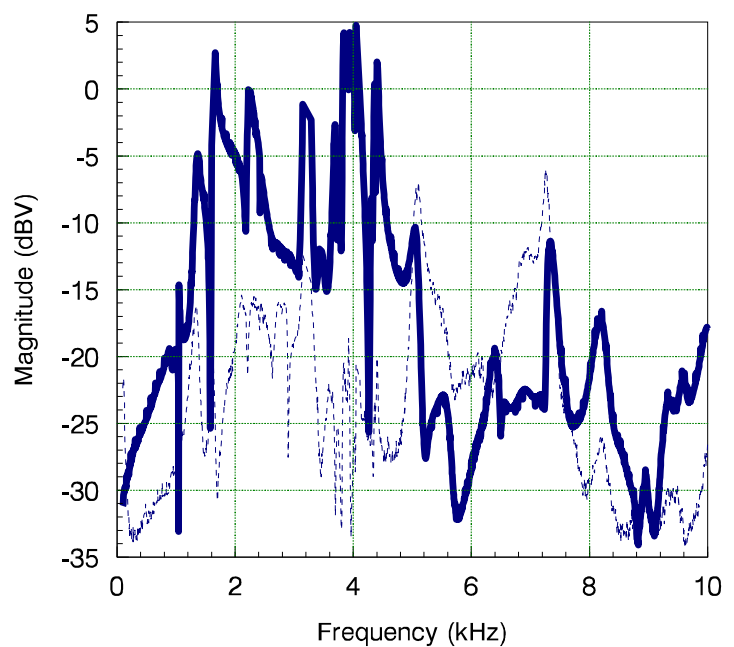

Fig. 4. Plot of the signal levels from the fixed and suspended transducers in response to vibrations in the baseplate as a function of frequency. Again the solidtrace is the signal from the fixed piezoelectric transducer, the light (dashed) trace is the suspended microphone.

we roll off the amplifiers in the sensor above $3 \mathrm{kHz}$.

\section{FIRMWARE}

The PIC12F683 microcontroller embedded in this device handles all of the computing tasks of the tamper unit. The firmware that is programmed onto this microcontroller is split into two functions, the interrupt triggered regularly by a timer and the main function that loops indefinitely.

The internal timer interrupt manages the microphone sam- 
pling, alarm decisions and pushbutton routine. Figure 5 shows a simplified flow chart of the internal timer interrupt.

The first step is to sample each of the microphones. This is performed with the built in analogue to digital converter in the microchip. A computationally cheap running average of the energy of each microphone is then computed and compared to operator defined thresholds. If the alarm conditions are met then an alarm flag is triggered, that is; the Piezo Microphone energy is greater than the Piezo threshold AND the Piezo Microphone energy is $x$ (operater defined) times the Free Microphone energy. In reality these steps are performed slighly out of synchronisation to maximise efficiency through performing operations while the analogue to digital convertor settles. This allows an $8 \mathrm{kHz}$ sample rate in each channel running at 4 MIPS on the microcontroller's internally-generated clock.

The pushbutton routine that enables an operator to set threshold values is also controlled within this interrupt. When pushbutton activity is detected we can temporarily disregard any alarm activity as this means there is currently an operator present at the unit.

The main loop simply performs the statistical logging and alarm handling functions. On detection of the alarm trigger flag the relay and LED are turned on.

In adition to the main workings of the unit, the prototype units have had statistical logging functions included in their firmware. Statitics such as piezo microphone events, microphone saturations, alarms, number of powerups and seconds of power applied are updated every 15 seconds while not currently in an event. A tally of alarms per quarter hour is also logged so data can be better analysed. These statistics are all stored in the non-volatile flash memory in the PIC12F683 chip.

In order to ensure a quick enough microphone sampling rate in order that no events are missed, the timer interrupt is called every $128 \mu \mathrm{s}$. The interupt function takes $90 \mu$ s to complete, meaning the program is interrupt dominated, and is only in the main loop for 30 percent of the time. However, this is not a problem as none of the vital operations are performed in the main loop, as explained above.

\section{Field Tests}

To determine how the units would function in the field several prototypes were deployed at various locations including bus shelters, road signs, roading barriers, doors, vending machines and a chain link fence. These locations involved a large variation of human activity. During the field testing, a small coin (weight 3.30 grams, slightly heavier than a US penny) was dropped from measured heights onto the object being monitored in order to measure sensitivity.

In the case of the steel roading barrier unit, the device was set as sensitive as possible (a drop of less than half an inch or $\approx 1 \mathrm{~cm}$ with the coin would set it off). This was sensitive enough so that without the inhibition process a passing vehicle would set the alarm off. However with the test units deployed no alarms were reported, despite this extreme sensitivity.

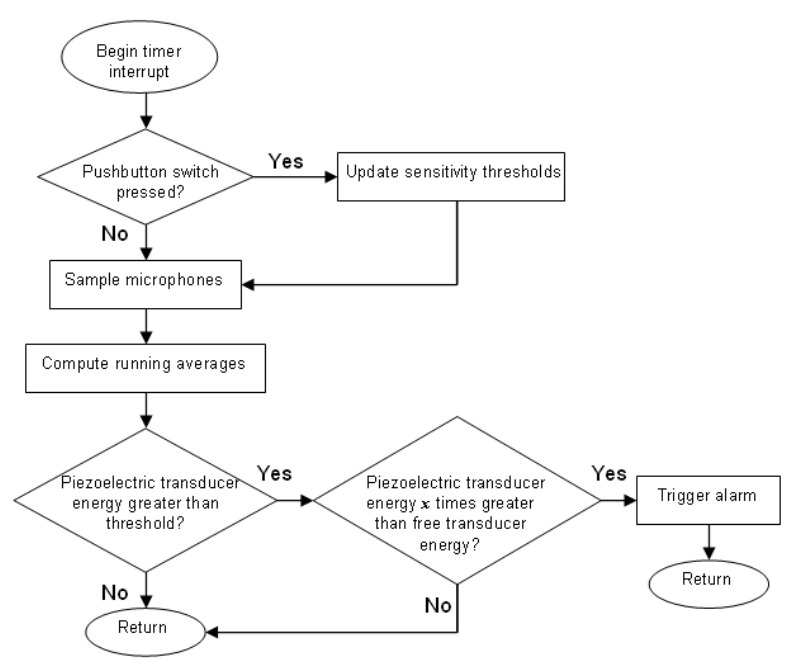

Fig. 5. A simplified flow chart of the timer interrupt used in the tampering detector.

An experiment was conducted with one of the bus shelter units. This unit was attached to the steel bench seat of the bus shelter and was set intially with a low enough sensitivity so without the inhibit process employed by our sensor, one would not expect to observe false alarms. This corresponded to a sensitivity that required roughly a 5 inch $(13 \mathrm{~cm})$ drop with the coin for an alarm. After two days in the field the statistics reported zero alarms. The same unit was then reinstalled with maximum sensitivity ( $<0.5$ inch or $\approx 1 \mathrm{~cm}$ coin drop). After two days the unit reported several alarms. This proved that it could pick up events (with an almost zero false alarm rate) where a similar unit without inhibition properties would produce numerous false alarms.

A unit was deployed on a chain link fence as part of a challenge that could be attempted by the general public [17], [18]. The fence was located in a public place where anybody could access it. This was a two part challenge-the first part being a prize for anyone who could successfully climb the fence without triggering an alarm. The second part of the challenge involved a more substantial prize for anyone who could set off the alarm without any physical impact on the fence (e.g. by means of loud noise, shaking the ground, etc).

Despite the challenge running for several weeks(??), no prizes were awarded. Climbing the fence proved to be extremely difficult due to the high sensitivity that could be achieved on the unit. Ultimately nobody was confident enough that they would be able to perform the task infront of an official in order to claim the prize. Triggering an alarm without touching the fence was also never achieved despite numerous attempts.

As a supplement to this challenge, loud noise tests were performed. The object of these tests were to saturate (overload) both microphones by a considerable amount and see how the unit behaved. The results showed that despite both microphones saturating their respective op-amps, the sensor behaves well. One channel registers more average energy than the other 
throughout the event. A massive impact on the unit shows the running average of the piezo microphone energy is greater than the running average of the free microphone the majority of the time. In this case the alarm is triggered, as alarm conditions only require the piezo microphone energy to exceed the free microphone energy for an instant. To test the reverse situation we used pyrotechnics to produce very loud sounds mechanically isolated from the unit. Recorded data shows that although both microphones saturate, throughout the event the average energy of the piezo microphone never exceeds the average energy of the free microphone. This therefore does not produce an alarm, the desirable outcome.

\section{CONCLUSION}

This tamper detector provides a new means of achieving excellent sensitivity without risking false alarms. It is cheap to manufacture, reliable and effective. During field testing these units showed outstanding results and have so far proved to be far superior to any other similar pieces of technology that are available today.

\section{REFERENCES}

[1] Gomery, R.; Leach, G. "Fence vibrations [intruder detection]", IEEE Aerospace and Electronic Systems Magazine, Volume 15, Issue 9, September 2000, pp:3-6.

[2] Bevan, G.; "Car alarms-origins to present systems and future trends", IEE Colloquium on Vehicle Security, 25 October, 1990 Pp:2/1-2/2.

[3] Jean-Claude Lahaye, "Noise Sensing Method And Intrusion Detection Device", European patent WO96/27862 A1, 1996.

[4] J. de Vries, "A low cost fence impact classification system with Neural Networks", IEEE AFRICON 2004

[5] Roy Baxendale, 'Intruder Alarm System", US patent 4,742,338, May 3, 1988.

[6] Mori Hideo, and Shibata Kiwamu, "Glass Breakage Sensor", Japan patent 2003-025030, publication JP2004-233300A, 2004.

[7] Eccardt et al., "Process For Monitoring The Windows Of A Room", European patent WO9206455A1, 1992.

[8] McNeilly et al., "Compentsated Intruder-Detection Systems", US patent 4,479,113, October 23, 1984.

[9] Ernst Spirig, "Ambient Noise Intruder Alarm", US patent 4,241,338, December 23, 1980.

[10] Harry F. Olson, "Acoustical Engineering", Van Nostrand, 1957.

[11] Robert E. Riley, Jr., Jackson, Miss. "Detection Systems", US patent 3,665,445, May 23, 1972.

[12] Raymond Tucker, "Detecting Persons Concealed in a Vehicle", International patent WO 2005/031671 A2, 7 April, 2005.

[13] R. Keith Harman, "Intrepid Update 1998", IEEE Carnahan Conference 1994

[14] Dr. Mel Maki, "Conductive Sensor Cables for Perimeter Intrusion Detection"

[15] M.E. Motamedi, "Acoustic Sensor Technology"

[16] John R. Vig, "On Acoustic Sensor Sensitivity" IEEE Transactions on Ultrasonics, Ferroelectrics and Frequency Control, Vol. 38. No. 3. May 1991

[17] Geoff Lewis, "Beat My Device, Challenges Professor", Hamilton Press, January 23, 2008, pp1-2.

[18] Jeff Neems, "Waikato group patents security system that prevents false alarm", The Waikato Times, January 26, 2008, page A10. 